\title{
Outcome of Cadmium on Aminergic System and Protective Function of Vitamin-C
}

\author{
Jyostna.V ${ }^{1}$, Anuhya.G ${ }^{1}$, Ushakiranmayi. Managamuri ${ }^{2}$, Srinuvasulu $\mathrm{K}^{3}$ and Sudhakar. Poda ${ }^{1}$ * \\ ${ }^{1}$ Department of Biotechnology, Acharya Nagarjuna University, Nagarjuna Nagar, Guntur, India \\ ${ }^{2}$ Department of Microbiology, Acharya Nagarjuna University, Nagarjuna Nagar, Guntur, India \\ ${ }^{3}$ Department of Biotechnology, K L University, Vaddeswaram, Guntur, India \\ *Corresponding author: sudhakarpodha@gmail.com, Phone No: +91-9000122929
}

Available online at: www.isroset.org

Received: 12/Mar/2018, Revised: 20/Mar/2018, Accepted: 14/Apr/2018, Online: 30/Apr/ 2018

\begin{abstract}
Cadmium (Cd) is an extremely toxic metal and has a long biological half-life in humans, it enters the brain parenchyma and neurons causing neurological alterations in humans and animal models, leading to lower attention, hyper nociception, olfactory dysfunction and memory deficits. Rats were exposed to low ( $2 \mathrm{mg} / \mathrm{kg} \mathrm{bw})$ and high dose (5mg/kg bw) of $\mathrm{Cd}$ through subcutaneous injections. The activity levels of epinephrine, norepinephrine and dopamine contents were studied in discrete brain areas of the young rats such as hippocampus, cerebral cortex and cerebellum. Hippocampal levels of catecholamines were significantly reduced in rats exposed to $\mathrm{Cd}$. Our data suggest that $\mathrm{Cd}$ exposure disturbs aminergic system in the hippocampus, cerebral cortex and cerebellum and may contribute to the alterations in neurotransmitters.
\end{abstract}

Keywords- Dopamine, Epinephrine, Norepinephrine, Cadmium, Vitamin-C

\section{Introduction}

Neurotransmitters such as Dopamine (DA), Epinephrine(EPI) and Norepinephrine(NE) contain catechol group or 1,2-dihydroxy benzene ring in their structure, and are called catecholamines and they are derived from the tyrosine by the catalytic action of the enzyme, tyrosine hydroxylase(TH) (1) having separate and distinct pathways in the brain. They are located in various brain regions and other tissues and serve many functions like emotion, attention and visceral regulation. DA is a biogenic amine neurotransmitter that has been shown to mediate central nervous systems (CNS), cardiovascular, renal and hormonal through the stimulation of alpha- adrenergic, betaadrenergic, and dopaminergic receptors (2) and it is an intermediate in the synthesis of norepinephrine and epinephrine and acts as neurotransmitter in the brain tissue and also influences the movement, behaviour and motor function. Norepinephrine acts as a transmitter by exerting most of its effects on post synaptic cells, but in adrenal medulla it acts as a hormone. A sensory stimulus alarms the animal and electrifies it into inclination by increasing the concentration of epinephrine in the blood and prepares the animal for emergency in several ways, increases the heart rate and the blood pressure to prepare the cardiovascular system for emergency action (3).
Vitamin- C (ascorbic acid) is an essential micronutrient required for normal metabolic functioning of the body (4). Humans and other primates cannot synthesize vitamin $\mathrm{C}$ as a result of a mutation in the gene coding for $\mathrm{L}$ gulonolactone oxidase, an enzyme required for the biosynthesis of Vitamin-C via the glucuronic acid pathway (5). It can be obtained through diet; lack of vitamin $C$ in the diet causes the deficiency disease scurvy (6). It is a cofactor for several enzymes involved in the biosynthesis of neurotransmitters, collagen and carnitine (7).

Vitamin-C is mainly helpful for catecholamine biosynthesis, in particular the conversion of dopamine to norepinephrine catalyzed by dopamine b-monooxygenase (8). Depression, hypochondria, and mood changes frequently occur during scurvy and could be related to deficient dopamine hydroxylation. Vitamin- $\mathrm{C}$ has ability to maintain metal ions in a reduced state which is related to its redox potential (9). In addition it is also helpful in maintenance of enzyme thiols in a reduced state and sparing of glutathione, an important intracellular antioxidant and enzyme cofactor and tetrahydrofolate, a cofactor required for catecholamine biosynthesis (10).

\section{Materials and methods}

Procurement and maintenance of animals 
The study was conducted to evaluate the antioxidant efficacy of Vitamin $\mathrm{C}$ against the $\mathrm{Cd}$ induced toxicity in young rats. Young (1month) male albino rats (Wistar) were purchased from National Institute of Nutrition, Hyderabad and maintained in the animal house of Nirmala college of pharmacy, Atmakur, Guntur. All the rats were kept in well cleaned, sterilized polypropylene cages lined with paddy husk (18" x 10" x 8"). The animals were maintained under a regulated light: dark $12 \mathrm{~h}$ (7:00-19:00) scheduled at $24 \pm$ $1^{\circ} \mathrm{C}$ and relative humidity of $55 \pm 15 \%$. Rats were provided standard rat chow (Sai Durga Feeds and Foods, Bangalore, India) and water ad libitum. The experiments were carried out in accordance with the guidelines of the CPCSEA1629/PO/a/12 and approved by the Institutional Animal Ethical Committee at Nirmala college of pharmacy of India (017/IAEC/NCPA 2016).

\section{Animal exposure}

After a week of acclimatization, young rats were randomly divided into five groups. Rats in the first group served as controls second and third group rats were exposed to a low dose of $2 \mathrm{mg} / \mathrm{kg}$ body weight and a high dose of $5 \mathrm{mg} / \mathrm{kg}$ body weight through subcutaneous injection for a period of four weeks. Rats of fourth and fifth group for were exposed to a low dose of $2 \mathrm{mg} / \mathrm{kg}$ body weight and a high dose of $5 \mathrm{mg} / \mathrm{kg}$ body weight through subcutaneous injection for 3 weeks and left for a period of one week for supplementation with Vitamin $\mathrm{C}$ at a dose of $50 \mathrm{mg} / \mathrm{kg}$ body weight through subcutaneous injection. Control animals received only saline without $\mathrm{Cd}$.

\section{Estimation of Catecholamines}

The levels of catecholamines (epinephrine, norepinephrine, and dopamine) were determined according to the method of (11). Synaptosomal fractions were taken in acid butanol to give a final concentration of $5 \mathrm{mg} / \mathrm{ml}$ and centrifuged at $800 \mathrm{~g}\left(-4^{0} \mathrm{C}\right)$ for 10 minutes. Residues were discarded and to the supernatant, $2.5 \mathrm{ml}$ of distilled water and $2.5 \mathrm{ml}$ of heptane were added. The contents were thoroughly mixed and centrifuged at $1000 \mathrm{~g}$ for 5 minutes. To the aqueous phase, $200 \mathrm{mg}$ of acid alumina was added followed by $1.5 \mathrm{ml}$, $2 \mathrm{M}$ sodium acetate. The contents were mixed thoroughly for 5 minutes and the $\mathrm{pH}$ was adjusted to 8.0 with $1 \mathrm{~N}$ sodium hydroxide.

The acid alumina was washed by vortexing the tubes twice with $2.0 \mathrm{ml}$ of distilled water and then centrifuged at $1000 \mathrm{~g}$ for 5 minutes. The supernatant was discarded and the walls of the tubes were blotted with strips of filter paper. The alumina was then vortexed for 5 minutes with $2.0 \mathrm{ml}$ of $0.2 \mathrm{~N}$ acetic acid to elute the catecholamines. The tubes were centrifuged at $1000 \mathrm{~g}$ for 5 minutes. The supernatants were transferred to $0.1 \mathrm{ml}$ of $0.1 \mathrm{M}$ EDTA and the $\mathrm{pH}$ was adjusted to 6.3. This was followed by the addition of $0.1 \mathrm{ml}$ of $0.1 \mathrm{~N}$ iodine solution. The tubes were mixed thoroughly and allowed to stand for two minutes. Then $0.2 \mathrm{ml}$ of alkaline sulphite solution was added. The contents were mixed and allowed to stand for 2 minutes at room temperature and the $\mathrm{pH}$ of the solution was adjusted to 5.4 with $5 \mathrm{~N}$ acetic acid. The samples with known amount of different amine standards were separately run to serve as internal standards. The fluorescence of epinephrine was read in a Spectrofluorometer with excitation and emission wavelengths of 410 and $500 \mathrm{~nm}$ respectively with a bandwidth of $10 / 10 \mathrm{~nm}$.

Norepinephrine was estimated by heating the same solution for 2 minutes in a boiling water bath. The tubes were cooled and the fluorescence of norepinephrine was read with excitation and emission wavelengths of 385 and $485 \mathrm{~nm}$ respectively with a slit width of $10 / 10 \mathrm{~nm}$. After the estimation of norepinephrine, the same solution was again heated for 5 minutes in a boiling water bath. Then the tubes were cooled and the fluorescence of dopamine was read with excitation and emission wavelengths of 320 and 370 $\mathrm{nm}$ respectively with a slit width of $10 / 10 \mathrm{~nm}$. The amine content of each tissue sample was calculated by the method of Ansell and Beeson (12) and expressed as $\mu \mathrm{g}$.amine/gm wet weight of tissue.

\section{Results and Discussion}

\section{Dopamine activity}

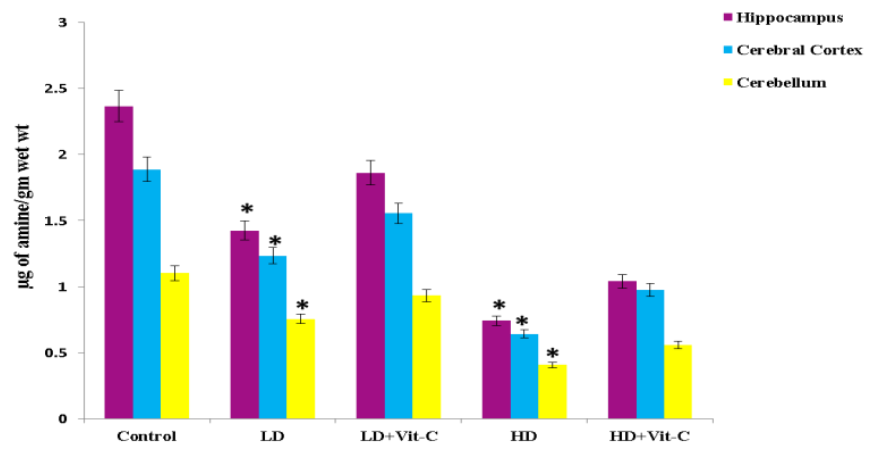

Figure 1 Effect of cadmium on Dopamine activity in brain regions of Hippocampus, Cerebral Cortex and Cerebellum of control and rats after exposure with cadmium at low dose(LD) (2mg) and high dose(HD) (5mg) through subcutaneous injections. The recovery of cadmium toxicity in separate group of albino rats exposed to cadmium subcutaneously at low and high dose treated with vitamin-C $(50 \mathrm{mg})(\mathrm{LDV}$ and HDV). All values are mean of values of six albino rats and values marked with(*) are significant at $\mathrm{P}<0.05-0.001$.

\section{Epinephrine activity}




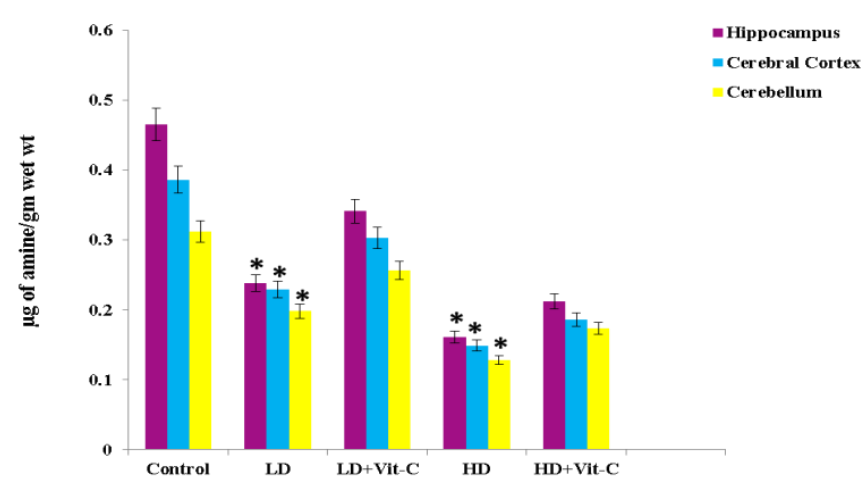

Figure 2: Effect of cadmium on Epinephrine activity in brain regions of Hippocampus, Cerebral Cortex and Cerebellum of control and rats after exposure with cadmium at low dose(LD) (2mg) and high dose(HD) (5mg) through subcutaneous injections. The recovery of cadmium toxicity in separate group of albino rats exposed to cadmium subcutaneously at low and high dose treated with vitamin-C (50mg)(LDV and HDV). All values are mean of values of six albino rats and values marked with $\left({ }^{*}\right)$ are significant at $\mathrm{P}<0.05-0.001$.

\section{Norepinephrine activity}

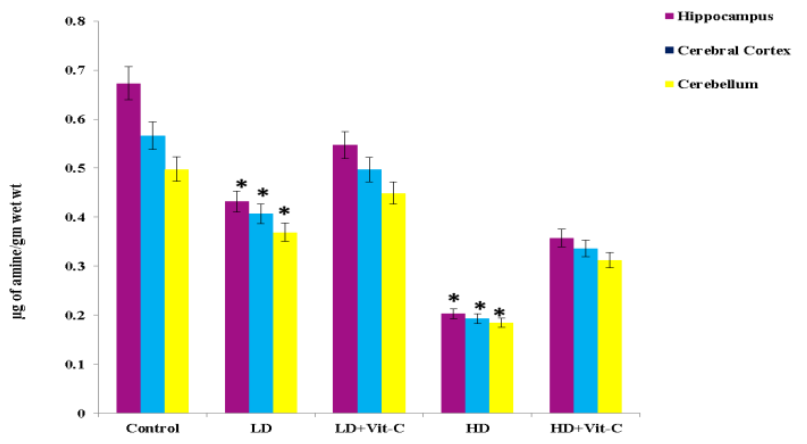

Figure 3: Effect of cadmium on Norepinephrine activity in brain regions of Hippocampus, Cerebral Cortex and Cerebellum of control and rats after exposure with cadmium at low dose(LD) (2mg) and high dose(HD) (5mg) through subcutaneous injections. The recovery of cadmium toxicity in separate group of albino rats exposed to cadmium subcutaneously at low and high dose treated with vitamin-C (50mg)(LDV and HDV). All values are mean of values of six albino rats and values marked with $(*)$ are significant at $\mathrm{P}<0.05-0.001$.

Our observation in the present study highlight that $\mathrm{Cd}$ administration has induced significant alterations in the levels DA, NE, and $E$ in various regions of rat brain such as cerebral cortex, cerebellum and hippocampus of both low and high dose substantiating that susceptibilities of different brain areas to $\mathrm{Cd}$ exposure could be related to local differences in their formation and maturation as well as the development of neurotransmitter systems might be affecting various steps in the metabolic pathway of the synthesis of these neurotransmitters. In addition to this, the administration of Vitamin-C along with $\mathrm{Cd}$ showed recovery from all synaptosomal aminergic system alterations in monoamine levels.

This neurotransmitters interact with each other in complex networks, for instance in the processes of learning and memory, in which dopamine and serotonin is proposed to have a fundamental role in locomotion $(13,14)$. Neurotransmitters are of interest as potential targets for neurotoxicants because they are integral to neuronal cell function and may play a role in neurite outgrowth. Since the catecholamines are thought to regulate motor activity in rodents, the levels of amines were among the first neurochemical parameters to be investigated in experimental $\mathrm{Cd}$ poisoning. Increasing evidence have shown that $\mathrm{Cd}$ accumulates in the brain is allied with changes in several neurotransmitter systems, as per studied in whole brain and in discrete brain areas (15). Chandra (16) stated that rats exposure to $\mathrm{Cd}$ has resulted variations in $\mathrm{DA}, \mathrm{NE}$ and serotonin (5-HT) in brain.

Neuromodulators such as norepinephrine and acetylcholine arise from brainstem and basal forebrain regions that have widespread projections throughout the brain, which are involved in memory consolidation and synaptic plasticity. Recent studies have illustrated that direct administration of epinephrine $(17,18)$ resulted significant improvements in memory. $\mathrm{Cd}$ administration not only induced alterations in catacheloamines but also it induces oxidative stress and increase in lipid peroxidation levels (LPO). Probably mechanism of $\mathrm{Cd}$ induced LPO is a contributing factor could be the enhancement of both DA release and turnover rate, as DA catabolism produces hydrogen peroxide $\left(\mathrm{H}_{2} \mathrm{O}_{2}\right)$ (19) and exogenous $\mathrm{H}_{2} \mathrm{O}_{2}$ decreased dopamine release by $50-60 \%$ (20). Various studies have been reported subsequently providing analysis of increased lipid peroxidation and oxidative stress as a result of Cd poisoning $(21,22)$.

Results of our present study showed that $\mathrm{Cd}$ exposure alters the aminergic system in synaptosomal catecholamine levels in the order of hippocampus, cerebral cortex and cerebellum. Several reports indicate that the hippocampus $(23,24)$ and nucleus accumbens (25) are important targets of this neurotoxic heavy metals such as lead and Cd. Andersson (26) have shown alterations in DA, 3,4-dihydroxyphenyl acetic acid (DOPAC), 5-HT, and 5HIAA content and metabolism in hippocampus, frontal cortex and striatum of rats exposed to Cd.

Cd even at low concentrations can disrupt a number of biological systems, that are much lower than most toxic metals $(27,28)$. It has the ability to increase the basal release 
of the neurotransmitters from the presynaptic nerve endings. This can occur both in the peripheral nervous system and central nervous system. This may be the reason for the observed decrease in the monoamine levels in synaptosomal fractions in the present study. Cd exposure is associated with an enhanced release of catecholaminergic neurotransmission based on the decreased levels of dopamine and norepinephrine, epinephrine.

$\mathrm{Cd}$ mostly accumulates in neural tissue in the central and peripheral nervous systems (29). Rats treated with different concentrations of $\mathrm{Cd}$ had more residues of $\mathrm{Cd}$ in multiple brain regions than control rats. In addition they also observed that prolonged exposure to relatively low amounts of $\mathrm{CdCl}_{2}$ results in cadmium accumulation in a dopamine-rich brain region (30). Our present findings are also parallel with these results that rats treated with $\mathrm{Cd}$ show more amount of $\mathrm{Cd}$ residues in brain regions than control.

Several reports indicate that the $\mathrm{Cd}$ can increase or decrease the basal concentration of dopamine in brain $(31,32)$. Besides, higher concentrations of $\mathrm{Cd}$ have been demonstrated to inhibit dopamine uptake in rat striatal synaptosomes, signifying that $\mathrm{Cd}$ intrinsically hinders the function of the dopamine transporter (33). Chronic $\mathrm{Cd}$ exposure also results in the loss of striatal neurons and a change in the number or function of vesicular monoamine and dopamine transporters, contributory role of cadmium toxicity in the development of neurological or psychiatric disorders associated with dysfunction of central dopaminergic pathways, such as schizophrenia or Parkinson's disease $(34,35)$. These results are also consistent with our results that $\mathrm{Cd}$ inhibits neurotransmitter amines such as DA, EPI and NE.

Ascorbic acid can influence the elaboration and functioning of nervous tissue and it is required for the transformation of dopamine into noradrenaline. Moreover, the biosynthesis of catecholamines occurs in brain and the adrenal gland in the presence of ascorbic acid (36). Dehydroascorbic acid is a oxidized form of ascorbic acid which can cross the blood brain barrier by means of facilitative transport and act as a neuroprotective agent in brain (37).Our results agrees with this statement that Vitamin- $\mathrm{C}$ reduces the $\mathrm{Cd}$ induced alteration in brain.

In our present study $\mathrm{Cd}$ administration with in short time duration resulted decrease in catecholamine concentration in young rats at both doses. Decreasing of catecholamine concentration in high dose is more than low dose. From this study we can illustrate that decrease in catecholamines is in dose dependent manner. The reason may be Tyrosine hydroxylase $(\mathrm{TH})$ is a rate limiting enzyme in the biosynthesis of catecholamine and catalyzes the first step of a biochemical synthetic pathway in which L-tyrosine is converted to L- 3, 4dihydroxy-phenylalanine (L-dopa). Recent studies reported that $\mathrm{PKC} \delta$ negatively regulates $\mathrm{TH}$ activity and dopamine synthesis by enhancing PP2A activity in dopaminergic neurons (Zhang et al., 2007). In addition to this decrease in DA content is also found in all brain regions of adult male and of newborn rats after $\mathrm{Cd}$ administration (Antonio et al., 2010).

\section{Conclusion}

From our present study we can allude that $\mathrm{Cd}$ exposure alters the monoamine levels in a dose dependent manner in all three rat brain regions. These alterations can be reversed with administration of Vitamin-C. Supplementation with antioxidant is more effective in combating with the $\mathrm{Cd}$ induced alterations on monoamine levels. However chelation with Vitamin-C helps in reducing the $\mathrm{Cd}$ burden effectively in $\mathrm{Cd}$ exposed rats.

\section{References}

[1] R. Shiman, M.Akino, S. Kaufman Solubilization and partial purification of tyrosine hydroxylase from bovine medulla. J. Biol. Cheer. 246, 1330-1340. 1971.

[2] M.Velasco, A.Lushsinger, Dopamine: Pharmacologic and Therapeutic Aspects. Am J Ther Vol 5, Issue 1, pp. 37-43. 1998.

[3] J.R.Cooper, F.E.Bloom, R.H. Roth, In the biochemical Basis of Neuropharmacology, $4^{\text {th }}$ edition. Oxford University Press, New York. 1982.

[4] G.M. Jaffe Vitamin C. In: Machlin L, ed. Handbook of vitamins. New York: Marcel Dekker Inc, pp.199-244. 1984

[5] A.A.Woodall, B.N. Ames Diet and oxidative damage to DNA: the importance of ascorbate as an antioxidant. In: Packer L, Fuchs J, eds. Vitamin C in health and disease. New York: Marcel Dekker Inc, pp.193-203. 1997.

[6] M. Levine New concepts in the biology and biochemistry of ascorbic acid. N Engl J Med Vol 314, pp.892-902. 1986.

[7] C.S. Tsao An overview of ascorbic acid chemistry and biochemistry. In: Packer L, Fuchs J, eds. Vitamin C in health and disease. New York: Marcel Dekker Inc, pp25-58. 1997.

[8] B.J.Burri, R.A. Jacob Human metabolism and the requirement for vitamin $\mathrm{C}$. In: Packer L, Fuchs J, eds. Vitamin C in health and disease. New York: Marcel Dekker Inc. pp.341-66. 1997.

[9] B.Halliwell Vitamin C: antioxidant or pro-oxidant in vivo. Free Radic Res. Vol25, pp. 439-54. 1996.

[10] A.Meister Glutathione-ascorbic acid antioxidant system in animals.J Biol Chem Vol 269, pp. 9397400. 1994.

[11] H.P. Kari, P.P.Davidson, H.H. Herbert, M.H. Kochbar, Effects of Ketoamine on brain monoamine levels in rats. Res. Comm. Chem. Path. Pharamacol. Vol 20, pp. 475-488. 1978. 
[12] G.B.Ansell, M.F. Beeson, A rapid and sensitive procedure for the combined assay of noradrenaline, dopamine and serotonin in a single brain sample. Anal. Biochem. Vol 23 pp. 196-206. 1968.

[13] R.J. Beninger, The role of dopamine in locomotor control. Brain Research Reviews. Vol 6, 173-196. 1983.

[14] C. Oberlander, B. Blaquière, J.F. Pujol, Distinct functions for dopamine and serotonin in locomotor behaviour: evidence using the 5-HT1 agonist RU 24969 in globus pallidus-lesioned rats. Neuroscience Letters. Vol 67, pp.113-118. 1986.

[15] M.Antonio, T.Antonio, I. Corpas, , and. M. L. Leret, Neurochemical changes in newborn rat's brain after gestational cadmium and lead exposure. Toxicology Letters. Vol 104, Issue (1-2), pp.1-9. 1999.

[16] S.V. Chandra, K.Kalia, T. Hussain, Biogenic amines and some metals in brain of cadmium-exposed diabetic rats. J Appl Toxicol 5:378-381. 1999.

[17] L.Cahill, M.T. Alkire, Epinephrine enhancement of human memory consolidation: interaction with arousal at encoding. Neurobiol Learn Mem. Vol 79, pp.194198. 2003.

[18] T.Moor, L.Mundorff, A. Bohringer, C. Philippsen, W.Langewitz, Reino, S.T. H. Schachinger, Evidence that baroreflex feedback influences long-term incidental visual memory in men. Neurobiol Learn Mem. Vol 84, pp.168-174. 2005.

[19] J.P.Kehrer, Free radicals as mediators of tissue injury and disease. Crit Rev Toxicol. Vol 23, Issue 1, pp.2148. 1993

[20] L. Chen, L. Liu, S.Huang, Cadmium activates the mitogenactivated protein kinase (MAPK) pathway via induction of reactive oxygen species and inhibition of protein phosphatases 2A and 5," Free Radical Biology and Medicine. Vol 45, Issue 7, pp1035-1044. 2008.

[21] D.Manca, A.C. Ricard, B.Trotter, G.Chevalier, , Studies of lipid peroxidation in rat tissues following administration of low and moderate doses of cadmium chloride, Toxicology Vol 67, pp. 303-323.1991.

[22] A.Shukla, G.S.Shukla, R.C. Srimal, Cadmium-induced alterations in blood brain barrier permeability and its possible correlation with decreased microvessel antioxidant potential in rat,"Human and Experimental Toxicology. Vol 15, Issue 5, pp.400-405. 1996.

[23] H.Wieg and L. Altmann, Neurophysiology aspects of hippocampal neurotoxicity. Neurotoxicology. Vol 15, pp. 451-458. 1994.

[24] M.Gutowski, L.Altmann, K.Sveinsson, H.Weigand, Postnatal development of synaptic plasticity in the CA3 hippocampal region of control and lead exposed wistar rats. Dev. Brain. Res. Vol 98, pp. 82-90. 1997.

[25] P.Devoto, G.Flore, A. Ibba, W.Fratta, L. Pani, Lead intoxication during intrauterine life and lactation but not during adulthood reduces nucleus accumbens dopamine release as studied by brain microdialysis. Toxicol. Lett. Vol 121, pp. 199-206. 2001.

[26] H.Andersson, K.Petersson-Grave, E.Lindqvist, Low level cadmium exposure of lactating rats causes alterations in brain serotonin levels in the offspring. Neurotoxicol Teratol. Vol 19, pp.105-115. 1997.

[27] A.Bernard. Renal dysfunction induced by cadmium: biomarkers of critical effects. Biometals Vol 17, pp. 519-23. 2004

[28] G.Nordberg K.Nogawa M,Nordberg L.Friberg Cadmium. In: Handbook on toxicology of metals.New York: Academic Press. pp. 65-78. 2007.

[29] B.Arvidson, Autoradiographic localization of cadmium in the rat brain. Neurotoxicology Vol 7 pp.89- 96. 1986.

[30] D.E.Clark, J.R.Nation, A.J. Bourgeois, M.F.Hare, D.M.Baker, E.J Hinderberger,. The regional distribution of cadmium in the brains of orally exposed adult rats. Neurotoxicology. Vol 6, 109-14. 1985.

[31] A.Lafuente, A.Gonzalez-Carracedo, A. Romero, A.I. Esquifino, Effect of cadmium on 24-h variations in hypothalamic dopamine and serotonin metabolism in adult male rats. Exp Brain Res. Vol 6, pp. 149:2002003.

[32] A.Lafuente, Marquez, N. D.Pazo, A.I. Esquifino, Effects of subchronic alternating cadmium exposure on dopamine turnover and plasma levels of prolactin, $\mathrm{GH}$ and ACTH. Biometals. Vol 13, pp. $47-55.2000$.

[33] J.C Lai,. L Lim, A.N.Davison, Differences in the inhibitory effect of $\mathrm{Cd}^{2+}, \mathrm{Mn}^{2+}$ and $\mathrm{Al}^{3+}$ on the uptake of dopamine by synaptosomes from forebrain and from striatum of the rat. Biochem Pharmacol. Vol 30, pp. 3123-3135. 1981.

[34] A.H. Evans, A.J.Lees, Dopamine dysregulation syndrome in Parkinson's disease. Curr Opin Neurol. Vol 17, Issue 393- 8. 2004.

[35] P.Remy, Y. Samson. The role of dopamine in cognition: evidence from functional imaging studies. Curr Opin Neurol. Vol 16 Issue 6, pp 37-41. 2003.

[36] M.Paleologos, R.Cumming and R.Lazarus Cohort study of vitamin $\mathrm{C}$ intake and cognitive impairment. Am. J. Epidemiol. Vol 148, pp 45-50. 1998;

[37] J.Huang, D.B.Agus, C.J. Winfree, S.Kiss, W.J.Mack, R.A.McTaggart, T.F. Choudhri, L.J.Kim, J.Mocco, D.J.Pinsky, W.D.Fox, R.J.Israel, T.A., Boyd, D.W. Golde, and E.S.Connolly, Jr: Dehydroascorbic acid, a bloodbrain barrier transportable form of vitamin $\mathrm{C}$, mediates potent cerebroprotection in experimental stroke. Proc Natl Acad Sci U S A Vol 98, pp.1172011724. 2001.

[38] D.Zhang, A. Kanthasamy, Y.Yang, V.Anantharam, A. Kanthasamy. Protein kinase cd negatively regulates tyrosine hydroxylase activity and dopamine synthesis by enhancing protein phosphatase- $2 \mathrm{a}$ activity in 
dopaminergic neurons. The Journal of Neuroscience. Vol 27, pp. 5349-5362. 2007.

[39] M.T. Antonio, V. Peinado, J.C. Gonza'lez, M.L Leret. Effects of maternal cadmium administration on development of monoaminergic, GABAergic and glutamatergic systems. Environ Toxicol Pharmacol Vol 29, pp. 87-90. 2010. 